\title{
Millimeter-wave GaN-based HEMT development at ETH-Zürich
}

\section{Journal Article}

Author(s):

Sun, Haifeng; Marti, Diego; Tirelli, Stefano; Alt, Andreas R.; Benedickter, Hansruedi; Bolognesi, Colombo R.

Publication date:

2010-04

Permanent link:

https://doi.org/10.3929/ethz-b-000026359

Rights / license:

In Copyright - Non-Commercial Use Permitted

Originally published in:

International Journal of Microwave and Wireless Technologies 2(1), https://doi.org/10.1017/S1759078710000164 


\title{
Millimeter-wave GaN-based HEMT development at ETH-Zürich
}

\author{
HAIFENG SUN, DIEGO MARTI, STEFANO TIRELLI, ANDREAS R. ALT, HANSRUEDI BENEDICKTER
}

AND C.R. BOLOGNESI

\begin{abstract}
We review the AlGaN/GaN high electron mobility transistor (HEMT) activities in the Millimeter-Wave Electronics Group at ETH-Zürich. Our group's main thrust in the AlGaN/GaN arena is the extension of device bandwidth to higher frequency bands. We demonstrated surprising performances for AlGaN/GaN HEMTs grown on high-resistivity (HR) silicon (111) substrates, and extended cutoff frequencies of $100 \mathrm{~nm}$ gate devices well into the millimeter (mm)-wave domain. Our results narrow the performance gap between GaN-on-SiC (or sapphire) and GaN-on-silicon and establish GaN-on-Si as a viable technology for low-cost mm-wave electronics. We here contrast the difference in behaviors observed in our laboratory between nominally identical devices built on high-resistivity silicon (HR-Si) and on sapphire substrates; we show high-speed devices with high-cutoff frequencies and breakdown voltages which combine $f_{T, M A X} \times B V$ products as high as 5-10 THz V, and show AlGaN/GaN HEMTs with $f_{T}$ values exceeding $100 \mathrm{GHz}$ on HR-Si. Although the bulk of our activities have so far focused on AlGaN/GaN HEMTs on HR-Si, our process produces excellent device performances when applied to GaN HEMTs on $\mathrm{SiC}$ as well: $100 \mathrm{~nm}$ gate transistors with $f_{T}>125 \mathrm{GHz}$ have been realized at ETH-Zürich.
\end{abstract}

Keywords: AlGaN/GaN, GaN-on-Si, HEMT, Millimeter-wave electronics

Received 15 November 2009; Revised 1 February 2010; first published online 20 April 2010

\section{INTRDDUCTIDN}

The present article highlights some of the AlGaN/GaN high electron mobility transistor (HEMT) activities of the recently established Millimeter-wave Electronics Laboratory at ETH-Zürich. The Group was launched in 2006 with the mandate to develop better, higher performance heterostructure devices, principally for future telecommunication systems. Our three principal areas of interest are double heterojunction bipolar transistors (DHBTs) based on the InP/GaAsSb heterostructure system, InP HEMTs for low-noise applications, and GaN-based HEMTs, which are the focus of the present article. The fundamental philosophy underlying our AlGaN/ GaN HEMT activities involves leveraging the ETH InP monolithically integrated circuit (MMIC) process capability to develop a GaN-based MMIC mm-wave platform.

Because of their now well-appreciated set of physical properties, GaN-based HEMTs remain the focus of intense interest for high-power, wideband, and/or high-temperature applications $[1,2]$. The key benefits of the (Al,Ga)N/GaN material system for microwave and mm-wave HEMTs are of course associated with their wide energy gaps and the high two DEG-channel charge densities resulting from the spontaneous and piezoelectric polarizations associated with the lattice mismatch between $(\mathrm{Al}, \mathrm{Ga}) \mathrm{N}$ and $\mathrm{GaN}$. In principle, this combination of characteristics makes possible rugged high-current drive wideband transistors capable of operating at high voltages and temperatures.

Millimeter-Wave Electronics Group, ETH-Zürich - Gloriastrasse 35, CH-8092 Zürich, Switzerland. Phone: +41446328775 .

Corresponding author:

C.R. Bolognesi

Email: colombo@ieee.org
The highest speed GaN HEMTs have historically been implemented on sapphire or on $\mathrm{SiC}$ substrates. Because of their affordability, ample supply, and good thermal conductivity at operating junction temperatures, high-resistivity silicon (HR-Si) substrates provide a low-cost solution for the realization of $\mathrm{GaN}$-based power transistors in the lower microwave frequency bands. Our group does not have a particular preference for any given substrate technology: historically, we have published results for devices grown on sapphire and SiC. More recently, since re-locating to ETH-Zürich, our work involved devices on HR-Si substrates because of a good relation with a supplier of epitaxial layers. As a result, our recent work established that GaN-based HEMTs grown on HR-Si also offer very good performances at mm-wave frequencies $[3,4]$, culminating with the realization of $100 \mathrm{~nm}$ gate $(\mathrm{Al}, \mathrm{In}) \mathrm{N} / \mathrm{GaN}$ HEMTs on HR-Si with cutoff frequencies as high as $f_{T}=102 \mathrm{GHz}$ for AlInN/GaN HEMTs grown at EPFL [5]. We will later show that these successes also carry over to devices on $\mathrm{SiC}$ substrates. All transistors discussed in this paper were fabricated on MOCVD-grown epitaxial layers.

In Section II, we compare the behavior of similar AlGaN/ GaN HEMTs grown on HR-Si and sapphire substrates. We show that although the devices display similar DC and RF performances, they react differently to various factors such as the presence of passivation layers. As well, we demonstrate highbreakdown voltages with interesting cutoff frequencies in transistors with larger source-to-drain spacings. In Section III, we discuss some the work our group has carried out to extend the operating frequencies of AlGaN/GaN HEMTs culminating with cutoff frequencies exceeding 100 and $125 \mathrm{GHz}$ for devices respectively grown on $\mathrm{HR}-\mathrm{Si}$ and $\mathrm{SiC}$ substrates. 
II. COMPARISON DF HEMTS $D N$ HR-SI AND SAPPHIRE SUBSTRATES

AlGaN/GaN heterostructures used for this study were MOCVD-grown on either (111) HR-Si or sapphire substrates. On HR-Si a $2 \mu \mathrm{m}$ GaN buffer was used, followed by $18.5 \mathrm{~nm}$ of $\mathrm{Al}_{0.27} \mathrm{Ga}_{0.73} \mathrm{~N}$ and $2 \mathrm{~nm} \mathrm{GaN}$ cap. A $2.9 \mu \mathrm{m} \mathrm{GaN}$ buffer was used on sapphire, followed by $25 \mathrm{~nm}$ of $\mathrm{Al}_{0.3} \mathrm{Ga}_{0.7} \mathrm{~N}$ and a $2 \mathrm{~nm} \mathrm{GaN}$ cap. The epitaxial layers are similar although not strictly identical, since they originate from different commercial vendors. Device processing details were given in [3]. The as-grown electron sheet densities and mobilities on HR-Si (sapphire) were, respectively, $8.5 \times 10^{12}\left(1.1 \times 10^{13}\right) /$ $\mathrm{cm}^{2}$ and $1200(1000) \mathrm{cm}^{2} / \mathrm{Vs}$.

Circular transmission line measurement patterns reveal sheet and contact resistance values of $640(550) \Omega /$ sq. and 0.46 (0.55) $\Omega \cdot \mathrm{mm}$ on the HR-Si (sapphire) substrate. DC characteristics were measured using an Agilent 4156A semiconductor parameter analyzer. Figure 1 superposes representative DC characteristics for $2 \times(0.1 \times 75) \mu \mathrm{m}^{2}$ devices before and after $\operatorname{SiN}_{x}$ passivation for devices built on the HR-Si substrate. The static output characteristics were measured for the range $V_{D S}=$ [o to 8] $\mathrm{V}$ with $V_{G S}=$ [o to -4$] \mathrm{V}$. The devices show similar output $I-V$ curves for both substrates, and a detailed comparison of $I-V$ characteristics was given in [6]. As expected, the drain saturation current increased after passivation: the drain current at $V_{G S}=0 \mathrm{~V}$ increased from $425 \mathrm{~mA} / \mathrm{mm}(500 \mathrm{~mA} / \mathrm{mm})$ to $545 \mathrm{~mA} / \mathrm{mm}(590 \mathrm{~mA} /$ $\mathrm{mm}$ ) on the HR-Si (sapphire) substrate. Figure 2 shows the transconductance characteristics before and after passivation for both substrates. We note that the threshold voltage shift toward more negative value following passivation. This may be due to an increase of sheet carrier concentration, but the phenomenon was not investigated in more depth. Peak DC extrinsic transconductances of 195 and $155 \mathrm{mS} / \mathrm{mm}$ were obtained for passivated devices on $\mathrm{HR}-\mathrm{Si}$ and sapphire substrates, respectively. Devices on HR-Si show a higher transconductance due to the thinner $(\mathrm{Al}, \mathrm{Ga}) \mathrm{N}$ barrier layer (18.5 nm on HR-Si versus $25 \mathrm{~nm}$ on sapphire). The dc transconductance values indeed scale roughly with the nominal barrier thicknesses, and the same ratio roughly holds for the values extracted from RF measurements. Devices built on sapphire showed current collapse-related instabilities before they were passivated by a $100 \mathrm{~nm}$ thick SiN film deposition, as shown in the transconductance plot of Fig. 2(b). The gate diode leakage current on the passivated devices of Figs 1 and 2 was measured at the level of 7.6 and $36 \mu \mathrm{A} / \mathrm{mm}$ at $V_{G}=-7 \mathrm{~V}$ for the HEMTs on HR-Si and sapphire, respectively. The lower gate leakage on HR-Si is attributable to the low screw dislocation densities $\left(\sim 10^{7} / \mathrm{cm}^{2}\right)$ achieved by Nitronex on their GaN-on-Si layers (see www.nitronex.com more information). The lower gate diode leakage achieved in our GaN on HR-Si HEMTs translates into better $I_{O N} / I_{O F F}$ ratios, as reported in [6].

$\mathrm{RF}$ measurements were performed from $\mathrm{DC}$ to $40 \mathrm{GHz}$ with an HP8510 vector network analyzer using an off-wafer calibration standard and an LRRM calibration. The effects of probing pads were de-embedded using open and short patterns. Figure 3 characterizes the transistor microwave performance of devices on HR-Si and sapphire substrate for bias points corresponding to the maximum transconductance with $V_{D S}=6 \mathrm{~V}$. Although the devices on both substrates display quite similar DC characteristics, they react very

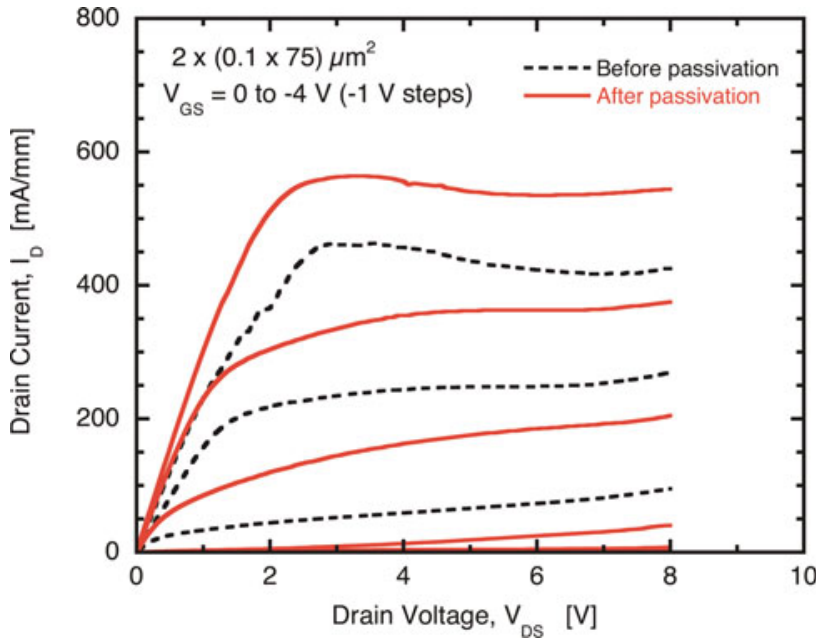

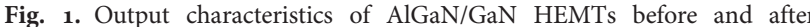
passivation on HR-Si substrate. Devices built on sapphire show nearly identical characteristics and are therefore not shown here since detailed comparison of their $I-V$ characteristics is given in [6].

differently in terms of microwave performance. A $-20 \mathrm{~dB} /$ dec extrapolation of $\left|h_{21}\right|^{2}$ and of Mason's maximum unilateral gain $U$ yields $f_{T}=49 / 56 \mathrm{GHz}$ and $f_{M A X}=90 / 108 \mathrm{GHz}$ before and after passivation for the devices on HR-Si: the passivation therefore markedly enhances the RF performance on HR-Si. For devices on sapphire, the $f_{T}$ and $f_{\text {MAX }}$ decrease from 57 to $54 \mathrm{GHz}$, and from 102 to $77 \mathrm{GHz}$, following passivation.

It may also be of interest to consider at the raw (or extrinsic) device performance with pad effects included. For the devices on HR-Si the pre-de-embedding cutoff frequencies are $f_{T, E}=38 / 46 \mathrm{GHz}$ and $f_{M A X, E}=73 / 88 \mathrm{GHz}$ before and after passivation, indicating that the $f_{M A X} / f_{T}$ ratio is essentially unaffected by the de-embedding process, thus contradicting the unfortunately too commonly held prejudice that GaN-on-Si devices necessarily suffer from conductive parasitic layers near the buffer/substrate interface. The parasitic buffer conduction can be satisfactorily addressed by optimizing growth conditions, as demonstrated here again on wafers from Nitronex. This view is strongly supported by our measurements of a CPW loss of only $0.82 \mathrm{~dB} / \mathrm{mm}$ at $110 \mathrm{GHz}$ for transmission lines built on HR-Si [6]. For devices built on sapphire, the pre-de-embedding cutoff frequencies are $f_{T, E}=43 / 44 \mathrm{GHz}$ and $f_{M A X, E}=81 / 63 \mathrm{GHz}$ before and after passivation. The role of passivation on the device RF performance is not straightforward when it comes to AlGaN/GaN HEMTs: an increase [7] as well as a decrease [8] of $f_{T}$ and $f_{\text {MAX }}$ after passivation have been reported by different groups. The present results provide additional food for thought since devices undergoing nominally identical process steps in the same laboratory show dramatically different behaviors, despite the resemblance in the initial epitaxial layer stacks. Clearly then, the area of passivation effects in AlGaN/GaN HEMTs is far from exhausted and much remains to be clarified.

Table 1 shows the difference in the small-signal parameters extracted from S-parameter measurements before and after passivation on both substrates. The ratio of $g_{M} / 2 \pi\left(C_{G S}+\right.$ $C_{G D}$ ) extracted from microwave measurements is in good agreement with the $f_{T}$ values obtained in Fig. 3. Our results 


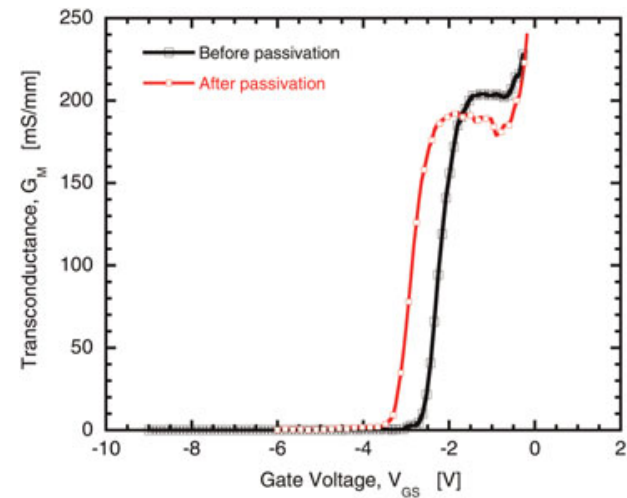

(a)

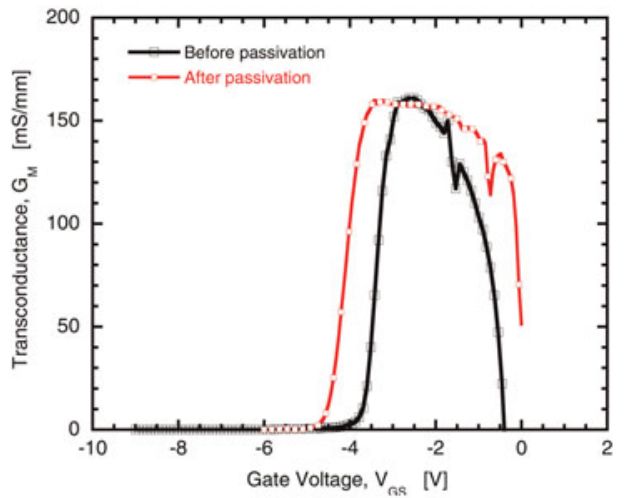

(b)

Fig. 2. Transconductance characteristics of AlGaN/GaN HEMTs before and after passivation: (a) on HR-Si substrate, and (b) Sapphire substrate. The unpassivated device on sapphire suffers current collapse which causes a drop of transconductance near $V_{G S}=0$ V. Passivation largely alleviates the current collapse on the devices built on sapphire.

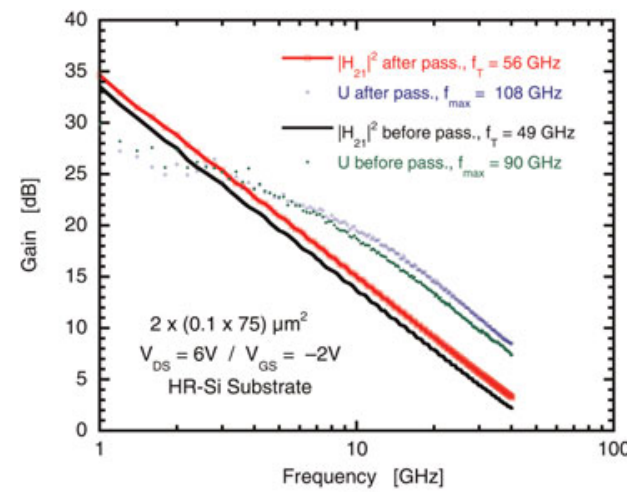

(a)

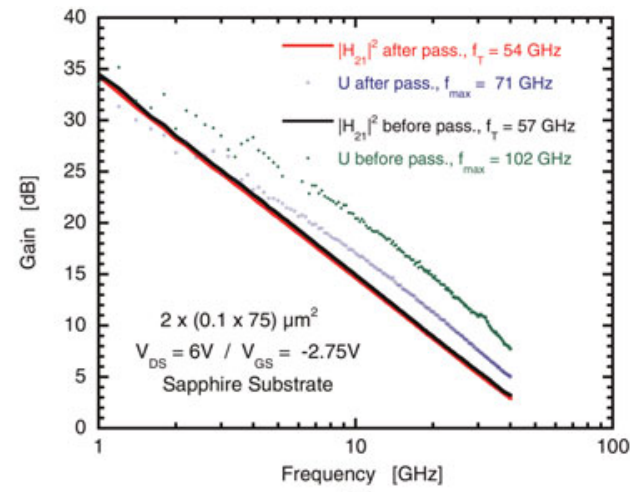

(b)

Fig. 3. Microwave performance of AlGaN/GaN HEMTs before and after passivation, with pad effects removed: (a) on the HR-Si substrate, and (b) on the Sapphire substrate.

show that the $\operatorname{SiN}_{x}$ passivation has higher impact on the parasitic capacitance (such as $C_{G S}$ and $C_{G D}$ ) for the GaN-on-sapphire devices than that on HR-Si. It should be mentioned that the passivated HEMTs built on HR-Si in fact feature a higher $f_{M A X} / f_{T}$ ratio compared to the passivated devices on sapphire, and that the higher $f_{M A X}$ can be traced to the lower microwave output conductance $g_{D S}$ on the HR-Si substrate [6].

In order to produce a high-gain millimeter-wave power amplifier featuring a high reliability, it is important to simultaneously achieve high transistor cutoff frequencies $f_{T}$ and $f_{M A X}$ and high breakdown voltages. GaN HEMTs have the potential to meet this requirement because the high electron velocities achieved under high electric fields can be combined with high breakdown voltage values achieved, thanks to the wide energy gaps of the materials involved. Although very high $f_{T} \times B V$ products were reported on sapphire and $\mathrm{SiC}$ [9], there is a scarcity of reported results for GaN-on-silicon device. Figure 4 characterizes the transistor microwave performance of devices on HR-Si with source-drain distance of $4 \mu \mathrm{m}$ : these devices were fabricated with a $0.1 \mu \mathrm{m}$ gate centered in the source-drain space. A $-20 \mathrm{~dB} / \mathrm{dec}$ extrapolation of $\left|h_{21}\right|^{2}$ and of Mason's maximum unilateral gain $U$ yields $f_{T}=43 \mathrm{GHz}$ and $f_{M A X}=77 \mathrm{GHz}$. Figure 5 shows the OFF-state breakdown voltage characterization of $B V_{D S}=110 \mathrm{~V}$ and $B V_{G D}=130 \mathrm{~V}$, as determined by the drain current injection technique of [10]. The $f_{T} \times B V$ products reach values as high as $5.6 \mathrm{THz}-\mathrm{V}$, and $10 \mathrm{THz}-\mathrm{V}$ when it comes to $f_{M A X} \times B V$. Still higher $f_{T, M A X} \times B V$ products could be achieved by positioning the gate closer to the source contact.

Transistors featuring high-cutoff frequencies and large breakdown voltages are not only attractive for high-efficiency high-power amplifier applications (which demand minimal dispersion non-idealities), but also in wideband highly rugged low-noise amplifiers which can be built without

Table 1. Device de-embedded parameters dependence on substrate type and passivation film.

\begin{tabular}{|c|c|c|c|c|c|c|c|c|c|}
\hline Substrate & Passivation & $f_{T}(\mathrm{GHz})$ & $f_{M A X}(\mathrm{GHz})$ & $f_{M A X} / f_{T}$ & $g_{M, R F}(\mathrm{mS})$ & $C_{G S}(\mathrm{fF})$ & $C_{G D}(\mathrm{fF})$ & $C_{D S}(\mathrm{fF})$ & $G_{D S}(\mathrm{mS})$ \\
\hline \multirow[t]{2}{*}{ HR-Si } & Before & 48.9 & 90 & 1.84 & 20.3 & 56.2 & 10.6 & 18.6 & 2.4 \\
\hline & After & 56 & 109 & 1.94 & 26.8 & 61.5 & 14.7 & 22.1 & $3 \cdot 3$ \\
\hline \multirow[t]{2}{*}{ Sapphire } & Before & 57 & 102 & 1.79 & $17 \cdot 5$ & 41.9 & 8.4 & 22.7 & 3.0 \\
\hline & After & 54 & 71 & 1.31 & 22.5 & 52.6 & 14.8 & 20.4 & 4.6 \\
\hline
\end{tabular}




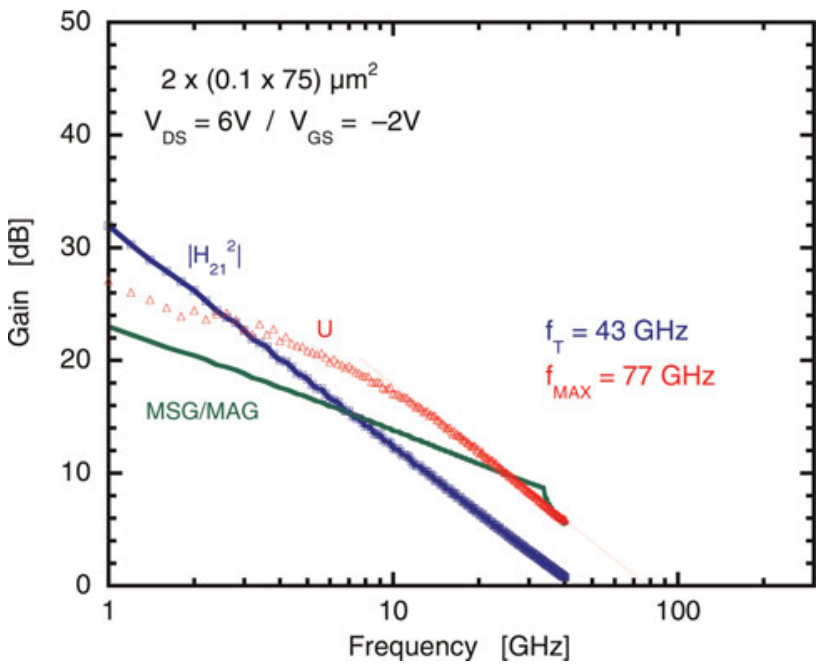

Fig. 4. Microwave performance measured at $V_{D S}=6 \mathrm{~V}$ and $V_{G S}=-2 \mathrm{~V}$ for $2 \times(0.1 \times 75) \mu \mathrm{m}^{2}$ HEMT. Extrapolation at $-20 \mathrm{~dB} / \mathrm{dec}$ yields $f_{T}=43 \mathrm{GHz}$ and $f_{\text {MAX }}=77 \mathrm{GHz}$. Pads effects de-embedded.

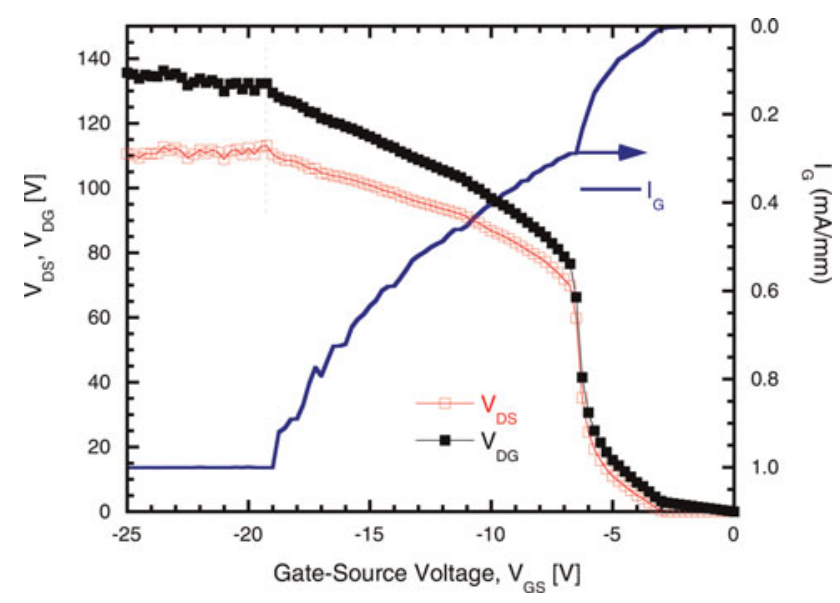

Fig. 5. Three terminal off-state breakdown characteristics of the conventional HEMT using the drain-current injection technique [10]. The drain current is fixed at $1 \mathrm{~mA} / \mathrm{mm}$ and $V_{G S}$ is swept from o to $-25 \mathrm{~V}$ (same device as Fig. 4).

input protection circuitry thanks to the low-noise figures achievable at X- and K-band frequencies [3]. In this context, it becomes interesting to push AlGaN/GaN HEMTs to higher frequencies of operation. This is also of interest for us at ETHZ.

\section{EXTENSION TOHIGHER FREQUENCIES}

Perhaps because of the unavailability of truly semi-insulating silicon substrates, comparatively little work has been performed prior to 2008 to determine whether or not AlGaN/ GaN HEMTs on silicon offer a viable technological solution for the realization of $\mathrm{mm}$-wave transistors with interesting properties. We have first reported very good cutoff frequencies with $f_{T}$ of $75 \mathrm{GHz}$ and noise figures interesting properties in $0.1 \mu \mathrm{m}$ HEMTs grown on HR-Si [3]. With increasing process maturity, we eventually achieved HEMTs with an $f_{T}$ of $90 \mathrm{GHz}$ [4]. On improved epitaxial layers featuring an electron mobility of $\sim 1500 \mathrm{~cm}^{2} / \mathrm{Vs}$ from Nitronex Corporation, we now fabricate fully passivated $(2 \times 75) \mu \mathrm{m}$ wide $(\mathrm{Al}, \mathrm{Ga}) \mathrm{N} / \mathrm{GaN}$ HEMTs with $100 \mathrm{~nm}$ gate lengths and source-drain spacing $L_{S D}$ of $1 \mu \mathrm{m}$. These devices feature an $f_{T}=104 \mathrm{GHz}$ which is the highest ever achieved for GaN-based HEMTs on silicon substrates, shown in Fig. 6. Although the bulk of our activities have so far focused on AlGaN/GaN HEMTs on HR-Si because of an excellent supply relationship with Nitronex, our process also results in attractive performances when applied to GaN HEMTs on $\mathrm{SiC}$ : Fig. 7 shows that $100 \mathrm{~nm}$ gate transistors with $f_{T}$ of $126 \mathrm{GHz}$ have been realized at ETH-Zürich. As far as we know, these are the fastest GaN-based HEMTs so far produced in Europe. More recently, we have also demonstrated the realization of $100 \mathrm{~nm}$ gate AlInN/GaN HEMTs with $f_{T}=144 \mathrm{GHz}$ and $f_{\text {MAX }}=145 \mathrm{GHz}$ using epitaxial layers

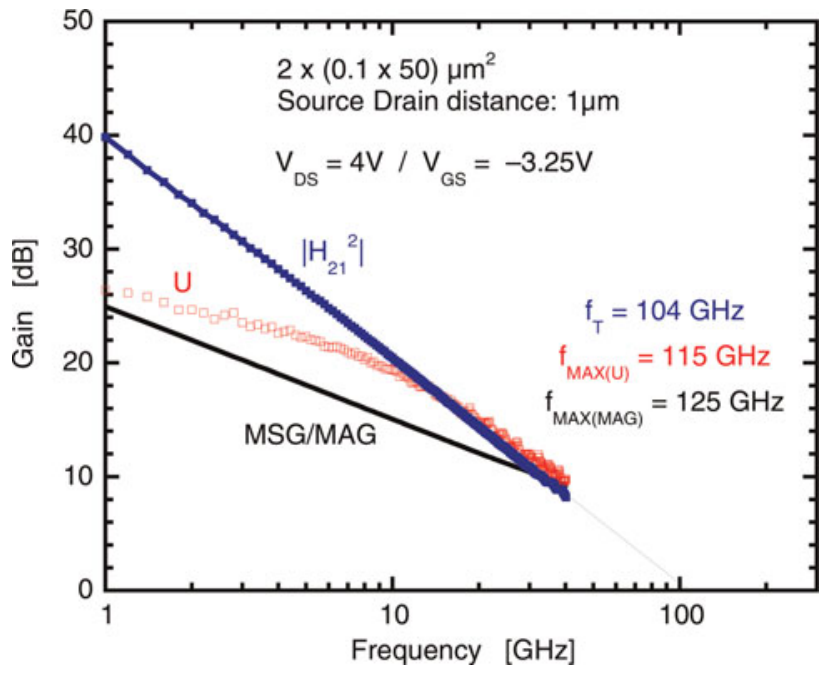

Fig. 6. Microwave performance measured at $V_{D S}=4 \mathrm{~V}$ and $V_{G S}=-3.25 \mathrm{~V}$ for $2 \times(0.1 \times 75) \mu \mathrm{m}^{2}$ HEMT on silicon substrate. Extrapolation at $-20 \mathrm{~dB} /$ dec yields $f_{T}=104 \mathrm{GHz}$ and $f_{M A X(U)}=115 \mathrm{GHz}$ and $f_{M A X(M A G)}=125 \mathrm{GHz}$. Pad effects de-embedded.

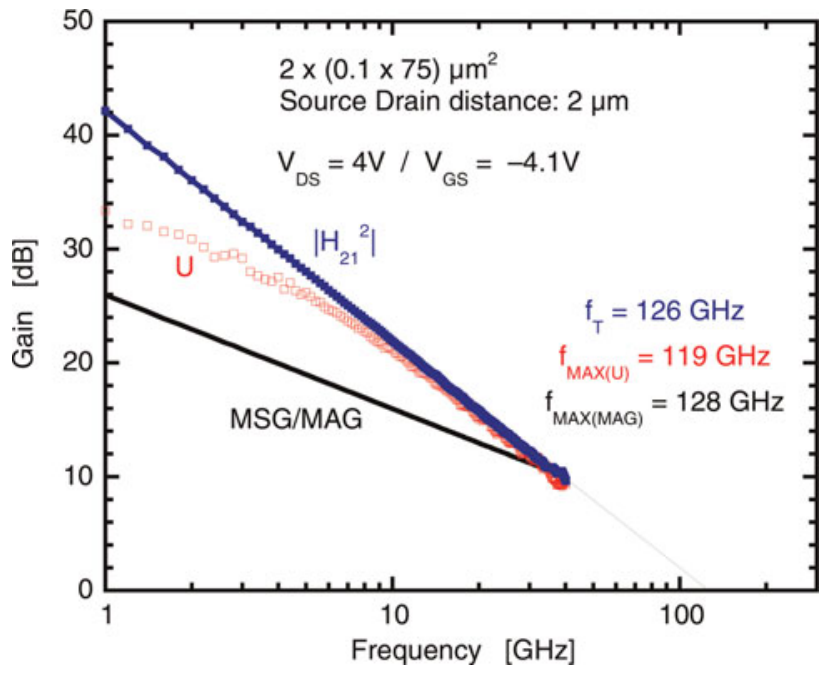

Fig. 7. Microwave performance measured at $V_{D S}=4 \mathrm{~V}$ and $V_{G S}=-4.1 \mathrm{~V}$ for $2 \times(0.1 \times 75) \mu \mathrm{m}^{2}$ HEMT on SiC substrate. Extrapolation at $-20 \mathrm{~dB} /$ dec yields $f_{T}=126 \mathrm{GHz}$ and $f_{M A X(U)}=119 \mathrm{GHz}$ and $f_{M A X(M A G)}=128 \mathrm{GHz}$. Pad effects de-embedded. 
grown on $\mathrm{SiC}$ at EPFL, thus setting a new record for AlInN/ $\mathrm{GaN}$ and closing the cutoff frequency gap between AlInN/ GaN and AlGaN/GaN HEMTs [11].

\section{SUMMARY}

Since being established in 2006, the Millimeter-Wave Electronics Group at ETH-Zürich has developed highperformance AlGaN/GaN HEMT processes enabling the demonstration of surprisingly good RF and noise performances for material grown on HR-Si (111) substrates, therefore narrowing the performance gap between devices grown on silicon and on insulating substrates such as sapphire and SiC. The ETHZ AlGaN/GaN HEMT process architecture is not limited to HR-Si substrates, as it also provides excellent device performances for materials grown on $\mathrm{SiC}$ substrates, as exemplified by the realization of $100 \mathrm{~nm}$ gate HEMTs exhibiting cutoff frequencies of $f_{T}=126 \mathrm{GHz}$ on $\mathrm{SiC}$ semiinsulating substrates. Work is currently underway to exploit these device performances by establishing a power MMIC process at ETH-Zürich.

More recently, our Group set the record for the highestspeed AlInN/GaN HEMT ever reported with an $f_{T}=$ $102 \mathrm{GHz}$ using EPFL material grown on HR-Si and demonstrated a reasonable output power of $2.5 \mathrm{~W} / \mathrm{mm}$ at $10 \mathrm{GHz}$ in $100 \mathrm{~nm}$ gate transistors built in a $1 \mu \mathrm{m}$ source-drain space which only allowed low breakdown voltages which limited the output power level [5]. As of writing time, we have now established a new record for $100 \mathrm{~nm}$ gate AlInN/ GaN HEMTs, again on EPFL material but this time grown on S.I. SiC to reach cutoff frequencies of $f_{T}=144 \mathrm{GHz}$, which matches the fastest achieved for thin-barrier AlGaN/ $\mathrm{GaN}$ devices of the same gatelength [11]. It is noteworthy that the fastest thin-barrier AlGaN/GaN HEMTs use thin $\sim 2 \mathrm{~nm}$ $\mathrm{SiN}$ passivation/gate dielectric films and also feature relatively leaky gates.

Although an increasing number of research workgroups worldwide have undertaken the development of nitride transistors for mm-wave applications, few if any have shown activities in this direction in Europe. This is, however, a role that naturally fits the interests and expertise of our Group, regardless of substrate type.

\section{ACKNDWLEDGEMENT}

The support of the FIRST Laboratory personnel at ETH is gratefully acknowledged.

\section{REFERENCES}

[1] Palacios, T. et al.: High-power AlGaN/GaN HEMTs for Ka-band applications. IEEE Electron Device Lett., 26 (2005), 781-783. doi: 10.1109/LED.2005.857701.

[2] Higashiwaki, M.; Mimura, T.; Matsui, T.: Millimeter-wave GaN HFET technology. Proc. SPIE, 6894 (2008), 68941L1-68941L9.

[3] Sun, H.F.; Alt, A.R.; Benedickter, H.; Bolognesi, C.R.: High-performance $0.1 \mu \mathrm{m}$ gate $\mathrm{AlGaN} / \mathrm{GaN}$ HEMTs on silicon with low-noise figure at $20 \mathrm{GHz}$. IEEE Electron Device Lett., 30 (2009), 107-109. doi: 10.1109/LED.2008.2010339.
[4] Sun, H.F.; Alt, A.R.; Benedickter, H.; Bolognesi, C.R.: 100-nm gate AlGaN/GaN HEMTs on silicon with $\mathrm{f}_{T}=90 \mathrm{GHz}$. Electron. Lett., 47 (2009), 376-377. doi: 10.1049/el.2009.0074.

[5] Sun, H.F.; Alt, et al.: 102-GHz AlInN/GaN HEMTs on silicon with 2.5-W/mm output power at $10 \mathrm{GHz}$. IEEE Electron Device Lett., 30 (2009), 796-798. doi: 10.1109/LED.2009.2023603.

[6] Sun, H.F.; Alt, A.R.; Marti, D.; Vetter, M.; Benedickter, H.; Bolognesi, C.R.: Small-signal microwave performance comparison of deep submicron AlGaN/GaN high electron mobility transistors on highresistivity silicon and insulating substrates. Appl. Phys. Express, 2 (2009), 111002-1-3. doi: 10.1143/APEX.2.111002-1-111002-3.

[7] Harrison, I.; Clayton, N.W.; Jeffs, N.J.: High temperature RF characterization of SiN passivated and unpassivated AlGaN/GaN HFETs. Phys. Status Solidi (a), 188 (2001), 275-278. doi: 10.1002/ 1521-396X(200111)188:1.

[8] Green, B.M.; Chu, K.K.; Chumbes, E.M.; Smart, J.A.; Shealy, J.R.; Eastman, L.F.: The effect of surface passivation on the microwave characteristics of undoped AlGaN/GaN HEMTs. IEEE Electron Device Lett., 21 (2000), 268-270. doi: 10.1109/55.843146.

[9] Kikkawa, T.; Imanishi, K.; Kanamura, M.; Joshin, K.: Recent progress of highly reliable GaN-HEMTs for mass production, in CS MANTECH Conf. Proc., April 2006, 171-174.

[10] del Alamo, J.A.; Somerville, M.H.: Breakdown in millimeter-wave power InP HEMT's: a comparison with GaAs PHEMT's. IEEE J. Solid-State Circuits, 34 (1999), 1204-1211. doi: 10.1109/4.782077.

[11] Sun, H.F. et al.: $100 \mathrm{~nm}$ Gate (Al,In)N/GaN HEMTs grown on $\mathrm{SiC}$ with $f_{T}=144 \mathrm{GHz}$. IEEE Electron Device Lett., 31 (2010), accepted for publication, 293-295.

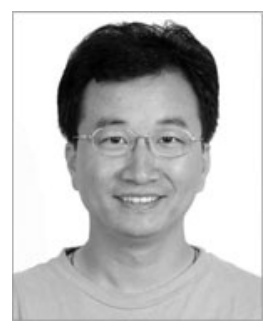

Haifeng Sun was born in Hunan, China. He received the B.S. degree in Applied Physics from Tsinghua University, Beijing, China, in 1997, and the M.S. degree in Electrical Engineering from the Institute of Microelectronics, Chinese Academy of Sciences, Beijing, China, in 2000. His Masters' thesis centered on the development of fully depleted SOI/CMOS devices and circuits. From 2001 to 2004, he joined Chinese Academy of Sciences, as an electrical engineer responsible for high-performance InGaP/ GaAs HBTs. He began working toward the Ph.D. degree at Simon Fraser University, Burnaby, BC, Canada, in September 2004. In 2006, he transferred to the Swiss Federal Institute of Technology (ETH), Zürich, Switzerland, where he is currently completing the Ph.D. degree in the Millimeter-Wave Electronics Group. His current research interests are the optimization and characterization of highperformance GaN-based devices.

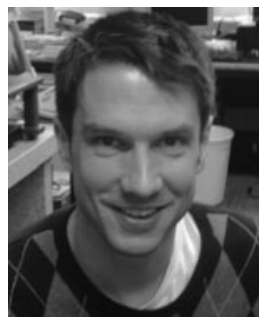

Diego Marti was born in Zürich, Switzerland. He received the M.Sc. in Electrical Engineering and Information Technology from the Federal Institute of Technology (ETH), Zürich, Switzerland, in 2009. His studies focused on circuit design and optoelectronics design and processing. For his Masters thesis, he designed and implemented wideband distributed amplifiers in a hybrid 
microstrip environment using $100 \mathrm{~nm}$ gate GaN HEMTs grown on high-resistivity silicon. Diego Marti started his Ph.D. work in 2009 at ETH and is working in the field of GaN HEMTs devices and circuit design and fabrication.

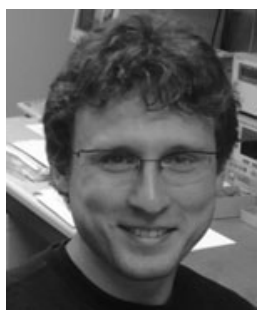

Stefano Tirelli was born in Reggio Emilia, Italy. He received the B.S. degree in Physics from University of Pisa, Italy in 2003, with a thesis carried out at California Institute of Technology, USA. He received his M.S. degree in Matter Physics from University of Pisa in 2008. His Masters' thesis, carried out at NEST Laboratory of Scuola Normale Superiore, Pisa, was focused on electronic transport in out-of-equilibrium superconductors. In January 2009 he moved to Switzerland to join the Millimeter-Wave Electronics Group at the Swiss Federal Institute of Technology (ETH), Zürich, where he is currently pursuing the Ph.D. His current research interests are related to the optimization of high-power GaN-based HEMTs and the investigation of electronic transport phenomena limiting the highfrequency performance in sub-100 $\mathrm{nm}$ gatelength devices.

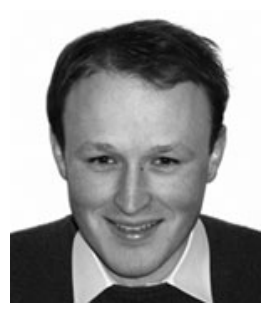

Andreas R. Alt was born in Munich, Germany. He received his B.S. degree in Electrical Engineering in 2004 and the Diploma (Masters) degree in Electrical Engineering in 2006 from Technical University of Munich (TUM), Germany. In 2006 he began his Ph.D. degree in the Millimeter-Wave Electronics Group at the Swiss Federal Institute of Technology (ETH), Zürich, Switzerland. His current research interests are the optimization/characterization and modeling of high-performance low-noise InP-based high electron mobility transistors.

Hansruedi Benedickter was born in Zug, Switzerland. He received the Diploma degree in Electrical Engineering from the Swiss Federal Institute of Technology (ETH), Zürich, Switzerland, in 1976. He has been a Research Assistant and a Senior Research Associate at the Microwave Laboratory, Swiss Federal Institute of Technology, and, since 1987, at the Laboratory for Electromagnetic Fields and Microwave
Electronics. His main research interests are microwave, millimeter-wave, and on-wafer measurement techniques.

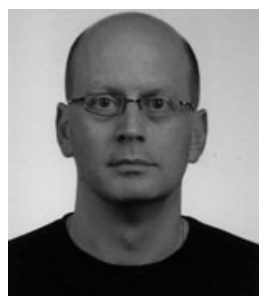

Colombo R. Bolognesi was born in St-Lambert QC, Canada. He earned the B.Eng. from McGill University (Montréal QC) in 1987, the M.Eng. from Carleton University (Ottawa ON) in 1989, and the Ph.D. from the University of California, Santa Barbara (UCSB) in 1993, all in Electrical Engineering. His doctoral dissertation dealt with the growth, fabrication, and physics of InAs/AlSb HFETs and led to the first high-speed InAs/AlSb HFET highyield process. In 1994, he joined Northern Telecom's Semiconductor Components Group in Ottawa, as a BiCMOS process integration engineer responsible for high-performance submicron polysilicon emitter BJTs with selectively implanted pedestal collectors. In 1995, he joined the Engineering Science and Physics Departments at Simon Fraser University to launch and direct SFU's Compound Semiconductor Device Fabrication Laboratory (CSDL). In 2001, his group demonstrated the first bipolar transistors in any material system with balanced $f_{T}$ and $f_{M A X}$ cutoff frequencies simultaneously exceeding $300 \mathrm{GHz}$ with a $6 \mathrm{~V}$ breakdown voltage, by exploiting the new InP/GaAsSb type-II heterojunction system. In 2006, he took up the Chair of Terahertz Electronics at the Institut für Feldtheorie und Höchstfrequenztechnik (IfH) at the Swiss Federal Institute of Technology in Zürich (ETH-Zürich). In 2010, the $\mathrm{THz}$ Electronics Group was renamed Millimeter-Wave Electronics and split from the IfH to stand as an independent Chair at ETH. Professor Bolognesi's research continues to target the development of high-speed devices (HBTs, HEMTs) based on InP/GaAsSb/ GaInAs and AlGaN/GaInN to extend their application toward higher frequencies. Among others, he and his team earned the IEEE 1999 GaAs IC Symposium Best Paper Award, and he was the co-recipient of the New Frontiers in Research Award from the Science Council of British Columbia in 2001. He serves/served on the Technical Program, Executive and/or International Steering Committees of several IEEE sponsored conferences such as the Device Research Conference (DRC'01 and '02), the Int. Electron Device Meeting (IEDM'O1,'O2, and '03 as the QEC Chair), and the Indium Phosphide and Related Materials Conference (IPRM'03, '07, '08, '09) as well as the Int. Symposium on Compound Semiconductors (ISCS’07, '08, '09). 